\title{
Progênies de seringueira com potencial para conferir resistência a ácaros (Calacarus heveae Feres e Tenuipalpus heveae Baker)
}

\author{
Rubber tree progenies with potential to confer resistance to the mite (Calacarus heveae Feres e \\ Tenuipalpus heveae Baker)
}

\author{
Marineide Rosa Vieira ${ }^{\mathrm{I}^{*}}$ Helder Adriano de Souza da Silva ${ }^{\mathrm{I}}$ Maria Monteverde Cardoso ${ }^{\mathrm{I}}$ \\ Janayna Correa FigueiraII
}

RESUMO

\begin{abstract}
Os ácaros Calacarus heveae Feres e Tenuipalpus heveae Baker são pragas importantes da cultura da seringueira. Dentre as opções de controle dessas pragas, o uso de material vegetal resistente pode ser uma boa estratégia. O presente trabalho teve por objetivo identificar progênies de seringueira com potencial para conferir resistência a essas duas espécies de ácaros, em uma área de progênies de polinização aberta, com comprovada variabilidade genética, para uso em programas de melhoramento. Os experimentos foram realizados em três períodos consecutivos, 2002/2003, 2003/ 2004 e 2004/2005, com avaliação de 33 progênies. A cada dois meses, de dezembro a junho do ano seguinte, foram coletados quatro ramos de cada planta, para contagem dos ácaros em laboratório. O nível de desfolhamento observado nas plantas foi avaliado com o uso de uma escala de notas de 0 (ausência de desfolhamento) a 4 (acima de 75\%). Das 33 progênies de seringueira testadas, oito apresentaram resistência por não preferência e/ou antibiose ou então por tolerância a pelo menos uma das espécies e deverão ser multiplicadas para futuras avaliações.
\end{abstract}

Palavras-chave: Hevea brasiliensis, Acari, Calacarus heveae, Tenuipalpus heveae.

\section{ABSTRACT}

The mites Calacarus heveae Feres e Tenuipalpus heveae Baker are important rubber tree pests. Among the control options, the resistant genetic material use can be a good strategy. The objective of this work was to identify rubber tree progenies with possibility of to confer resistance to these species in an area of open-pollinated progenies, with confirmed genetic variability, for use in genetic improvement program. The experiments were carried out in three consecutive periods, 2002/ 2003, 2003/2004 and 2004/2005 with evaluation of 33 progenies. Every two months, of December to June of the next year, were collected four branches of each plant for mites counting in laboratory. The defoliation levels observed were evaluated with a note scale of 0 (without defoliation) to 4 (above 75\%). Eight progenies showed non preference and/or antibiosis resistance or tolerance to at least one species and should be multiplied for new evaluations in the future.

Key words: Hevea brasiliensis, Acari, Calacarus heveae, Tenuipalpus heveae.

\section{INTRODUÇÃO}

Dentre as espécies de ácaros fitófagos que podem colonizar a cultura da seringueira [Hevea brasiliensis (Willd. ex Adr. de Juss.) Müell. Arg.], Calacarus heveae Feres (Eriophyidae) e Tenuipalpus heveae Baker (Tenuipalpidae) são as que têm causado os sintomas mais significativos nas áreas de cultivo comercial. A primeira delas tem sido detectada em altas populações, na face superior de folhas maduras de seringueira e, como resultado do seu ataque, as folhas perdem o brilho e apresentam um amarelecimento progressivo de sua superfície (VIEIRA et al., 2000). Plantas atacadas podem perder acima de $75 \%$ das suas folhas em um ou dois meses antes do desfolhamento natural (VIEIRA \& GOMES, 1999). T. heveae ocorre na face inferior das folhas, localizando-se ao longo das nervuras, onde é possível observar grande quantidade de ácaros, ovos e exúvias, com um escurecimento do tecido vegetal em correspondência aos locais de alimentação. As folhas atacadas tornam-se amareladas e posteriormente caem (VIEIRA \& GOMES, 2003).

\footnotetext{
IDepartamento de Fitossanidade, Engenharia Rural e Solos, Universidade Estadual Paulista (UNESP), CP 31, 15385-000, Ilha Solteira, SP, Brasil. E-mail: marineid@bio.feis.unesp.br. *Autor para correspondência.

II In morian.
} 
Dentro da perspectiva do manejo integrado de pragas, o uso de material vegetal resistente pode ser uma importante estratégia de controle, por reduzir os custos de produção e as possibilidades de contaminação do homem e do ambiente. Os programas de melhoramento da seringueira têm procurado avaliar produção, vigor, crescimento do caule durante a sangria, espessura da casca virgem, regeneração de casca, tolerância à queda pelo vento, tolerância à seca do painel e resistência às principais doenças (GONÇALVES, 1999). Porém, a resistência a pragas, insetos e ácaros não tem sido contemplada.

Informações sobre resistência a pragas, dentro de um programa de melhoramento genético para essa cultura, podem ser geradas a partir da avaliação de clones já desenvolvidos. Contudo, as avaliações podem começar em uma fase anterior, com a obtenção de sementes de polinização aberta ou controlada, cujas mudas serão levadas a campo para avaliação, e o sucesso depende da variação genética disponível na população (FURLANI, 2001).

Plantas resistentes aos ácaros que atacam a cultura podem ser detectadas em função de um menor desenvolvimento populacional das espécies, indicativo da presença de fatores de resistência por não preferência ou por antibiose. Mesmo no caso de uma grande infestação da praga, a planta pode apresentar resistência por tolerância, resultando em poucos danos (LARA,1991). No Brasil, o desenvolvimento populacional dos ácaros em diferentes clones comerciais de seringueira tem sido avaliado em alguns levantamentos de campo nos Estados do Mato Grosso (FERLA, 2001; DAUD \& FERES, 2007) e de São Paulo (FURQUIM, 1994).

Considerando os benefícios do uso de plantas resistentes a pragas, o presente trabalho teve por objetivo identificar genótipos com potencial para conferir resistência às espécies de ácaros-praga da cultura da seringueira, em uma área de progênies de polinização aberta, com comprovada variabilidade genética, para uso em programas de melhoramento genético.

\section{MATERIAL E MÉTODOS}

A área experimental localiza-se na Fazenda de Ensino, Pesquisa e Extensão da Universidade Estadual Paulista (UNESP), Campus de Ilha Solteira, no Município de Selvíria, Mato Grosso do Sul (MS) (latitude de $20^{\circ} 22^{\prime} \mathrm{S}$, longitude $51^{\circ} 22^{\prime} \mathrm{W}$ e altitude média de 335m). Essa área foi estabelecida em 06 de dezembro de 1991, sendo constituída por 420 plantas provenientes de sementes coletadas em uma população natural localizada em Rio Branco, Estado do Acre, originando progênies de polinização aberta, com comprovada variabilidade genética (FURLANI, 2001).

Os experimentos foram realizados em três períodos consecutivos, 2002/2003, 2003/2004 e 2004/ 2005, sempre de dezembro a agosto do ano seguinte. As plantas amostradas foram escolhidas entre as que apresentaram o maior perímetro do caule, indicativo de um bom desenvolvimento das plantas. No período 2002/ 2003, foram selecionadas e marcadas 17 plantas e, no segundo período, 27 foram escolhidas, sendo 11 do período anterior e 16 de uma nova seleção. Seis plantas do primeiro período não puderam mais ser avaliadas em função da elevada altura da copa. No período 2004/ 2005, 13 plantas que apresentaram resultados promissores foram avaliadas novamente.

De cada planta foram coletados quatro ramos de pontos diferentes da copa, utilizando-se uma tesoura de poda alta. Os ramos colhidos foram acondicionados em sacos plásticos e levados ao laboratório de Acarologia da UNESP, Campus de Ilha Solteira, para avaliação. As coletas foram realizadas, para cada período, nos meses de dezembro, fevereiro, abril e junho.

Uma vez que cada planta precisa ser avaliada individualmente porque se constitui em um material genético diferente dos demais, nos três experimentos, considerou-se um delineamento inteiramente casualizado, sendo cada planta um tratamento e cada ramo uma repetição.

Em laboratório, de cada ramo, foram retirados cinco folíolos, para contagem de $\boldsymbol{C}$. heveae, na página superior, e outros cinco folíolos, para contagem de T. heveae, na página inferior. Para o eriofídeo (C. heveae), foi realizada avaliação em duas áreas de $1 \mathrm{~cm}^{2}$, em cada folíolo, uma de cada lado da nervura principal e, para o ácaro vermelho (T. heveae), em duas áreas de $1 \mathrm{~cm}^{2}$ por folíolo, uma sobre a nervura principal e uma sobre uma nervura lateral.

Em cada período, os dados de número médio de ácaros por $10 \mathrm{~cm}^{2}$ foram transformados em log (x + 2), por não apresentarem aderência à normalidade (teste de Lilliefors). Depois de transformados, foram submetidos à análise de variância, e as médias foram agrupadas pelo teste de Scott-Knott, a 5\% de probabilidade. As infestações observadas nas 13 progênies, em 2004/2005, foram comparadas às registradas em 2003/2004, nos mesmos materiais, com a realização de uma análise de variância para cada espécie de ácaro.

O nível de desfolhamento observado nas plantas foi avaliado com o uso de uma escala de notas de 0 a 4, sugerida por VIEIRA \& GOMES (1999), sendo 
0 para ausência de desfolhamento, 1 para 0 a 25\% de desfolhamento, 2 para 25 a 50\%, 3 para 50 a $75 \%$ e 4 para desfolhamento acima de $75 \%$. Essas avaliações foram realizadas mensalmente, de dezembro a agosto do ano seguinte.

As progênies estudadas foram classificadas em três categorias, de acordo com o tipo e grau de resistência apresentados (LARA, 1991). Assim, foram consideradas resistentes por não preferência e/ou antibiose as progênies com número médio de ácaros agrupado entre as menores médias, em todos os períodos em que foram avaliadas (no mínimo dois) e com nota de desfolhamento igual ou inferior a 2,0 em todos os períodos (no mínimo dois). As progênies com número médio de ácaros agrupado entre as maiores médias em pelo menos um dos períodos considerados, mas com nota de desfolhamento igual ou inferior a 2,0, em todos os períodos (no mínimo dois), foram consideradas resistentes por tolerância. Por fim, foram consideradas suscetíveis as progênies com nota de desfolhamento superior a 2,0 em pelo menos um dos períodos avaliados. Nos casos em que a progênie apresentou baixa população de ácaros ou pouco desfolhamento, mas só foi avaliada em um único período devido à grande altura da copa, os resultados foram considerados inconclusivos.

\section{RESULTADOS E DISCUSSÃO}

Houve diferença significativa entre as progênies quanto ao desenvolvimento populacional dos dois ácaros considerados, expresso pelo número médio de ácaros registrado em cada período (Tabela 1).

Em 2002/2003, nove progênies apresentaram menores populações de $\boldsymbol{C}$. heveae $(\mathrm{F}=2,108 ; \mathrm{P}<0,01)$ e oito foram menos favoráveis ao ácaro T. heveae $(\mathrm{F}=$ 3,082; $\mathrm{P}<0,01$ ). Das plantas avaliadas, 13 apresentaram nota média de desfolhamento igual ou inferior a 2,0, portanto com menos de $50 \%$ da copa sem folhas.

Para o período 2003/2004, das 27 progênies estudadas, 14 apresentaram menores populações de C. heveae ( $\mathrm{F}=3,75 ; \mathrm{P}<0,01)$ e 15 menor desenvolvimento de T. heveae ( $\mathrm{F}=5,185 ; \mathrm{P}<0,01)$. Para 15 delas, a nota de desfolhamento foi igual ou inferior a 2,0.

Das 13 progênies avaliadas em 2004/2005, em nove, $\boldsymbol{C}$. heveae apresentou menor desenvolvimento ( $\mathrm{F}=8,55 ; \mathrm{P}<0,01)$ e cinco foram menos favoráveis ao ácaro T. heveae $(\mathrm{F}=10,309 ; \mathrm{P}<0,01)$. Oito materiais receberam notas de desfolhamento iguais ou menores que 2,0 .

Neste trabalho, o nível médio de desfolhamento de $50 \%$, nota 2,0, foi escolhido como o limite para a seleção dos materiais. Para o planalto paulista, a seringueira, espécie de hábito decíduo, apresenta senescência e reenfolhamento no período de julho a setembro (GONÇALVES et al., 2001). De acordo com CORTEZ \& MARTIN(1996), a produção de coágulo pela cultura da seringueira, no Estado de São Paulo, segue um padrão sazonal, com um crescimento a partir de setembro, um período de maior produção de janeiro a julho (com pico em maio/junho), decrescendo a partir daí até um valor mínimo em agosto/ setembro. Os ácaros, no presente trabalho, foram registrados nas coletas de fevereiro, abril e junho, portanto coincidindo com o período mais significativo da produção da cultura. Embora até o momento não existam informações sobre o efeito do desfolhamento na produção de látex, é provável que a queda antecipada de folhas deva resultar em redução no volume de látex produzido. Assim, plantas que apresentaram, ao longo do período, nota média de desfolhamento inferior a 2,0, embora também tenham desfolhado no final do ciclo, permaneceram com uma copa saudável por um tempo maior, característica que deve favorecer a produção.

Parte do desfolhamento observado pode ter ocorrido em função de um processo natural de senescência. Entretanto, ao longo dos três períodos, foi observada grande quantidade de folíolos com sintomas das duas espécies de ácaros nas plantas e no chão da área, indicando ser essa a principal causa do desfolhamento.

Quatro progênies apresentaram menores populações de $\boldsymbol{C}$. heveae nos três períodos, e, em FRP/ 832 e FRP/3511, o desfolhamento médio não ultrapassou $50 \%$, nota 2,0. Nesses dois materiais, as populações de T. heveae estiveram entre as maiores em 2002/2003 e 2004/2005, o que, entretanto, não resultou em maior queda de folhas. Ambos foram considerados resistentes aos ácaros, por não preferência e/ou antibiose ao ácaro $\boldsymbol{C}$. heveae e por tolerância ao ácaro T. heveae. Por outro lado, nas progênies FRP/2535 e FRP/2612, apesar das menores populações de $C$. heveae nos três períodos, o desfolhamento em 2004/ 2005 foi acima de 2,0, sendo classificadas como suscetíveis.

Para T. heveae, duas progênies apresentaram menores populações nos três períodos, FRP/924 e FRP/2515. Em 2002/2003, esses materiais foram agrupados com as maiores médias para número de ácaros de $\boldsymbol{C}$. heveae, mas receberam a menor nota de desfolhamento, 1,25, o que pode significar a presença de fatores de resistência por tolerância. No caso da FRP/924, essa hipótese foi confirmada nos dois períodos seguintes, nos quais, apesar das maiores populações do eriofídeo, as notas de desfolhamento 
Tabela 1 - Número médio de ácaros ${ }^{1}$ por $10 \mathrm{~cm}^{2}$, das espécies $\boldsymbol{C}$. heveae $(\boldsymbol{C h})$ e $\boldsymbol{T}$. heveae (Th), nota de desfolhamento, grau e tipo de resistência em progênies de seringueira. Selvíria-MS.

\begin{tabular}{|c|c|c|c|c|c|c|c|c|c|c|c|}
\hline \multirow{3}{*}{ Progênie } & \multicolumn{3}{|c|}{-------Período 2002/03------- } & \multicolumn{3}{|c|}{-------Período 2003/04-------- } & \multicolumn{3}{|c|}{-------Período 2004/05-------- } & \multicolumn{2}{|c|}{----Resistência ${ }^{3}$---- } \\
\hline & \multicolumn{2}{|c|}{$\mathrm{N}^{\mathrm{o}}$ de indivíduos } & \multirow[b]{2}{*}{ Nota } & \multicolumn{3}{|c|}{$\mathrm{N}^{\mathrm{o}}$ de indivíduos } & \multicolumn{2}{|c|}{$\mathrm{N}^{\mathrm{o}}$ de indivíduos } & \multirow[b]{2}{*}{ Nota } & & \\
\hline & Ch & Th & & Ch & Th & Nota & Ch & Th & & Ch & Th \\
\hline FRP/425 & $6,9 \mathrm{~b}^{2}$ & $1,9 \mathrm{~b}$ & 2,00 & & & & & & & SC & SC \\
\hline FRP/615 & $6,7 \mathrm{a}$ & $0,7 \mathrm{a}$ & 1,37 & $3,4 \mathrm{~b}$ & $3,5 \mathrm{a}$ & 2,21 & & & & $\mathrm{~S}$ & $\mathrm{~S}$ \\
\hline FRP/619 & $24,9 \mathrm{~b}$ & $0,7 \mathrm{a}$ & 1,81 & & & & & & & SC & SC \\
\hline FRP/725 & $3,1 \mathrm{a}$ & $1,2 \mathrm{~b}$ & 2,00 & $0,7 \mathrm{a}$ & $8,3 \mathrm{~b}$ & 2,36 & & & & $\mathrm{~S}$ & $\mathrm{~S}$ \\
\hline FRP/832 & $1,6 \mathrm{a}$ & $2,8 \mathrm{~b}$ & 1,50 & $0,6 \mathrm{a}$ & $0,5 a$ & 1,36 & 5,9 a & $3,2 \mathrm{~b}$ & 1,92 & $\mathrm{R}_{\mathrm{NP} / \mathrm{A}}$ & $\mathrm{R}_{\mathrm{T}}$ \\
\hline FRP/924 & $21,1 \mathrm{~b}$ & 0,6 a & 1,25 & $12,6 \mathrm{~b}$ & 1,7 a & 1,00 & $66,6 \mathrm{~b}$ & 0,9 a & 1,83 & $\mathrm{R}_{\mathrm{T}}$ & $\mathrm{R}_{\mathrm{NP} / \mathrm{A}}$ \\
\hline FRP/1615 & 4,9 a & 0,0 a & 2,06 & & & & & & & SC & SC \\
\hline FRP/1638 & $2,4 \mathrm{a}$ & $1,4 \mathrm{~b}$ & 1,44 & & & & & & & SC & $\mathrm{SC}$ \\
\hline FRP/2415 & $13,9 \mathrm{~b}$ & $0,8 \mathrm{a}$ & 1,75 & $7,5 \mathrm{~b}$ & $9,5 \mathrm{c}$ & 2,00 & 20,7 a & $31,0 \mathrm{c}$ & 2,25 & $\mathrm{~S}$ & $\mathrm{~S}$ \\
\hline FRP/2421 & 8,3 b & $2,4 \mathrm{~b}$ & 2,25 & $1,4 \mathrm{a}$ & $2,6 \mathrm{a}$ & 2,57 & & & & $\mathrm{~S}$ & $\mathrm{~S}$ \\
\hline FRP/2512 & $13,7 \mathrm{~b}$ & 0,6 a & 1,56 & $6,4 \mathrm{~b}$ & 2,7 a & 2,71 & & & & S & $\mathrm{S}$ \\
\hline FRP/2515 & $14,9 \mathrm{~b}$ & 0,6 a & 1,25 & 3,7 a & 3,6 a & 1,71 & $1,6 \mathrm{a}$ & $1,0 \mathrm{a}$ & 2,33 & S & $\mathrm{S}$ \\
\hline FRP/2535 & $2,4 \mathrm{a}$ & $1,1 \mathrm{a}$ & 1,50 & $0,9 \mathrm{a}$ & $3,6 \mathrm{~b}$ & 1,86 & 8,9 a & $4,6 \mathrm{~b}$ & 2,25 & $\mathrm{~S}$ & $\mathrm{~S}$ \\
\hline FRP/2612 & 20,6 a & $2,7 \mathrm{~b}$ & 1,56 & $0,9 \mathrm{a}$ & $1,7 \mathrm{a}$ & 1,71 & $2,4 \mathrm{a}$ & 1,1 a & 2,17 & $\mathrm{~S}$ & $\mathrm{~S}$ \\
\hline FRP/2723 & 4,7 a & $2,3 \mathrm{~b}$ & 2,37 & & & & & & & $\mathrm{~S}$ & $\mathrm{~S}$ \\
\hline FRP/3322 & $6,3 \mathrm{~b}$ & $2,5 \mathrm{~b}$ & 2,37 & & & & & & & $\mathrm{~S}$ & $\mathrm{~S}$ \\
\hline FRP/3511 & $2,1 \mathrm{a}$ & $2,0 \mathrm{~b}$ & 1,81 & $0,0 \mathrm{a}$ & $1,2 \mathrm{a}$ & 1,43 & $3,3 \mathrm{a}$ & $6,6 \mathrm{~b}$ & 2,00 & $\mathrm{R}_{\mathrm{NP} / \mathrm{A}}$ & $\mathrm{R}_{\mathrm{T}}$ \\
\hline FRP/216 & & & & $1,3 \mathrm{a}$ & $4,1 \mathrm{~b}$ & 2,00 & & & & SC & SC \\
\hline FRP/224 & & & & 10,7 b & 7,7 b & 3,00 & & & & $\mathrm{~S}$ & $\mathrm{~S}$ \\
\hline FRP/817 & & & & $4,8 \mathrm{~b}$ & $6,1 \mathrm{~b}$ & 1,79 & & & & SC & SC \\
\hline FRP/833 & & & & $1,0 \mathrm{a}$ & $1,9 \mathrm{a}$ & 2,14 & & & & $\mathrm{~S}$ & $S$ \\
\hline FRP/915 & & & & $2,1 \mathrm{a}$ & $0,4 \mathrm{a}$ & 2,43 & & & & S & $\mathrm{S}$ \\
\hline FRP/1611 & & & & $6,3 \mathrm{~b}$ & 0,8 a & 1,21 & $160,9 \mathrm{~b}$ & $1,5 \mathrm{a}$ & 1,67 & $\mathrm{R}_{\mathrm{T}}$ & $\mathrm{R}_{\mathrm{NP} / \mathrm{A}}$ \\
\hline FRP/1616 & & & & $13,1 \mathrm{~b}$ & $5,1 \mathrm{~b}$ & 2,64 & & & & S & $\mathrm{S}$ \\
\hline FRP/2435 & & & & 4,9 b & $12,7 \mathrm{c}$ & 2,36 & & & & S & $\mathrm{S}$ \\
\hline FRP/2531 & & & & $2,1 \mathrm{a}$ & 5,9 c & 1,36 & $6,6 \mathrm{a}$ & 9,7 c & 2,33 & $\mathrm{~S}$ & $\mathrm{~S}$ \\
\hline FRP/2812 & & & & $1,1 \mathrm{a}$ & $3,7 \mathrm{~b}$ & 2,43 & & & & $\mathrm{~S}$ & $\mathrm{~S}$ \\
\hline FRP/2821 & & & & $21,3 \mathrm{~b}$ & $0,4 \mathrm{a}$ & 1,57 & $34,1 \mathrm{~b}$ & $3,0 \mathrm{~b}$ & 1,75 & $\mathrm{R}_{\mathrm{T}}$ & $\mathrm{R}_{\mathrm{T}}$ \\
\hline FRP/3012 & & & & $4,0 \mathrm{~b}$ & $2,3 \mathrm{a}$ & 1,43 & $41,9 \mathrm{~b}$ & $17,2 \mathrm{c}$ & 1,75 & $\mathrm{R}_{\mathrm{T}}$ & $\mathrm{R}_{\mathrm{T}}$ \\
\hline FRP/3112 & & & & 17,6 b & $3,9 \mathrm{~b}$ & 2,57 & & & & $\mathrm{~S}$ & $\mathrm{~S}$ \\
\hline FRP/3514 & & & & $1,2 \mathrm{a}$ & 0,9 a & 2,50 & & & & $\mathrm{~S}$ & $\mathrm{~S}$ \\
\hline FRP/3523 & & & & $5,7 \mathrm{~b}$ & $5,3 \mathrm{~b}$ & 1,43 & 4,7 a & $3,4 \mathrm{~b}$ & 1,75 & $\mathrm{R}_{\mathrm{T}}$ & $\mathrm{R}_{\mathrm{T}}$ \\
\hline FRP/12110 & & & & $0,2 \mathrm{a}$ & $0,0 \mathrm{a}$ & 1,64 & $2,4 \mathrm{a}$ & 0,1 a & 2,00 & $\mathrm{R}_{\mathrm{NP} / \mathrm{A}}$ & $\mathrm{R}_{\mathrm{NP} / \mathrm{A}}$ \\
\hline
\end{tabular}

${ }^{1}$ Médias originais. Análise estatística realizada com os dados transformados em $\log (\mathrm{x}+2) .{ }^{2} \mathrm{Na}$ vertical, médias não ligadas por mesma letra diferem pelo teste de Scott-Knott, a $5 \%$ de probabilidade. ${ }^{3} \mathrm{~S}$ : suscetível; $\mathrm{R}_{\mathrm{NP} / \mathrm{A}}$ : resistência por não preferência e/ou antibiose; $\mathrm{R}_{\mathrm{T}}$ : resistência por tolerância; SC: sem conclusão.

estiveram abaixo de 2,0. Para a FRP/2515, nos dois períodos seguintes, houve uma menor população dos dois ácaros, mas, apesar disso, em 2004/2005, a nota de desfolhamento foi 2,33, sendo classificada como suscetível.

Na classificação das progênies quanto a possíveis fatores de resistência, alguns critérios foram considerados. Em primeiro lugar, a resistência é um caráter genético e, dessa forma, deve haver repetibilidade do comportamento observado (LARA,
1991). Assim, no presente trabalho, uma progênie foi considerada resistente por não preferência e/ou antibiose quando o número médio de ácaros foi agrupado entre as menores médias, em todos os períodos em que ela foi avaliada, caracterizando, assim, a repetibilidade da resistência. Também foi considerada resistente a planta que manifestou tolerância à presença dos ácaros, com nota de desfolhamento igual ou inferior a 2,0 em todos os períodos, apesar de o número médio de ácaros ter sido agrupado com as maiores 
médias. Por outro lado, quando a nota de desfolhamento foi superior a 2,0, a progênie foi classificada como suscetível. Materiais considerados suscetíveis foram descartados das avaliações seguintes.

Algumas progênies, embora com bons resultados na primeira avaliação, não puderam ser incluídas nas avaliações seguintes devido à altura da copa, muito acima da altura viável para corte. Esses materiais, mesmo com baixa infestação de ácaros e pouco desfolhamento, não foram considerados resistentes, uma vez que foram avaliados uma única vez. Nesses casos, os resultados foram considerados inconclusivos. Entretanto, permanecem como uma possibilidade para pesquisas futuras caso seja possível realizar a coleta foliar.

Entre os materiais avaliados em dois períodos, a progênie FRP/12110 não registrou a ocorrência de T. heveae em 2003/2004 e teve o registro de um único ácaro no mês de junho de 2005, média de 0,1 ácaros $10 \mathrm{~cm}^{-2}$ ao longo do período. O número médio de $\boldsymbol{C}$. heveae foi agrupado entre os menores, e a nota de desfolhamento foi de no máximo 2,0. Esse material foi classificado como resistente por não preferência e/ ou antibiose às duas espécies.

Em FRP/3012, foram registradas altas infestações dos dois ácaros em 2004/2005, mas, apesar disso, com nota média de desfolhamento de 1,75. Na avaliação de junho, realizada no dia 16 , das treze progênies avaliadas no período, quatro ainda permaneciam com a nota 1 (até $25 \%$ de desfolhamento), entre elas a FRP/3012, indicando a presença de possíveis fatores de resistência por tolerância. Baixo desfolhamento, apesar das maiores populações de $\boldsymbol{C}$. heveae, também foi observado nas progênies FRP/2821 e FRP/1611. Em 2004/2005, o nível populacional do eriofídeo nesses três materiais atingiu 4,2, 3,4 e 16,1 ácaros $\mathrm{cm}^{-2}$, respectivamente. VIEIRA \& GOMES (1999) observaram, para o clone RRIM 600, ocorrência de desfolhamento das plantas com níveis acima de 0,94 ácaros $\mathrm{cm}^{-2}$. Os três foram considerados resistentes por tolerância ao ácaro $C$. heveae, e os dois primeiros também foram considerados resistentes ao ácaro $\mathbf{T}$. heveae. FRP/1611 apresentou resistência por não preferência e/ou antibiose ao ácaro vermelho.

Na progênie FRP/1615, avaliada apenas em 2002/2003, não foi registrada a presença de T. heveae. Esse é um material importante para futuras avaliações.

A resistência de uma planta ao desenvolvimento populacional de uma praga pode ser atribuída a um comportamento de não preferência para alimentação, oviposição ou abrigo ou a um efeito de antibiose, quando a planta afeta a biologia da praga (LARA, 1991). No presente trabalho, não é possível determinar qual dos dois tipos de resistência está envolvido na resposta observada, ou mesmo se os dois participam do processo. Apesar de não possuírem asas, que lhes permitam optar pelo local adequado para pouso, os ácaros, depois de caírem em uma planta, transportados pelo vento, podem decidir entre ficar naquele hospedeiro e desenvolver uma colônia, ou buscar novas alternativas, pelo caminhamento ou assumindo comportamentos específicos de dispersão. Os eriofídeos, como é o caso de $\boldsymbol{C}$. heveae, podem elevar a parte anterior do corpo na direção do vento, para facilitar o processo de dispersão (SABELIS \& BRUIN, 1996). Dessa forma, a não aceitação de uma progênie como alimento ou abrigo pode ter levado a um comportamento de fuga. Por outro lado, o efeito de antibiose também é possível, uma vez que a presença de substâncias deletérias no substrato alimentar pode afetar o potencial reprodutivo dos ácaros (TADMOR et al., 1999; VIEIRA et al., 2002).

Progênies que apresentaram nota média de desfolhamento igual ou inferior a 2,0, apesar de níveis maiores de infestação dos ácaros, foram consideradas resistentes por tolerância. Esse tipo de resistência é caracterizado pelo desenvolvimento de poucos danos, apesar de uma grande infestação da praga (LARA, 1991). Das progênies avaliadas nos três períodos, cinco delas apresentaram nota acima de 2,0 somente na terceira avaliação. Esse fato pode ser explicado, pelo menos em parte, pela maior infestação verificada no terceiro período, tanto para $\boldsymbol{C}$. heveae $(\mathrm{F}=26,6 ; \mathrm{P}<0,01)$, quanto para T. heveae $(\mathrm{F}=13,62 ; \mathrm{P}<0,01)$. O nível de infestação é um dos fatores que podem afetar a manifestação da resistência (LARA, 1991) e, assim, uma planta resistente pode passar a exibir os danos da praga a partir de um nível de infestação maior. A partir disso, essas cinco progênies com nota acima de 2,0 em 2004/ 2005 foram consideradas suscetíveis.

A maior infestação registrada em 2004/2005 provavelmente esteve relacionada com a ocorrência de maiores precipitações, pelo menos com relação ao ácaro $\boldsymbol{C}$. heveae. Essa espécie é favorecida por ambiente úmido, conforme os dados de FERLA \& MORAES (2003), os quais verificaram, em estudos de laboratório, a viabilidade da fase de ovo a adulto acima de $90 \%$, com umidade relativa do ar de $90 \pm 5 \%$. O terceiro período de avaliação foi o mais chuvoso, com um total de precipitação, de outubro a junho do ano seguinte, de 1207 mm, em comparação com 997mm, em 2002/2003, e 904mm, em 2003/2004. Para T. heveae, PONTIER et al. (2000) observaram mortalidade de $67 \%$ na fase de ovo a adulto para ácaros mantidos em umidade relativa do ar de $70 \pm 10 \%$, sugerindo ser a alta umidade um fator prejudicial à espécie. Entretanto, DAUD \& FERES 
(2007), em Itiquira, Mato Grosso (MT), observaram pico populacional da espécie associado a períodos chuvosos para a região. Nesse caso, deve-se considerar que as precipitações naquele local foram menos intensas quando comparadas com as que ocorrem normalmente em época chuvosa no Estado de São Paulo. No caso desse ácaro, o efeito das condições climáticas permanece como um ponto para pesquisas futuras.

Nos experimentos realizados, foram selecionadas oito progênies com potencial para conferir resistência às duas espécies estudadas. A FRP/12110 foi classificada como resistente por não preferência e/ ou antibiose aos dois ácaros, devido às baixas infestações registradas nos dois períodos estudados. FRP/924 e FRP/1611 apresentaram resistência por não preferência e/ou antibiose ao ácaro T. heveae e tolerância ao ácaro $\boldsymbol{C}$. heveae. O inverso foi registrado para FRP/832 e FRP/3511, que foram tolerantes ao ácaro vermelho. Por último, as progênies FRP/2821, FRP/3012 e FRP/3523 apresentaram tolerância às duas espécies acarinas.

Esses oito materiais são interessantes para a realização de clonagem e acompanhamento do desenvolvimento das mudas e da produção. As progênies FRP/832 e FRP/924 já foram clonadas, e as mudas estão sendo avaliadas. Além destas, a progênie FRP 1615, avaliada apenas em 2002/2003, apresenta interesse para inclusão em novos estudos, em função de não ter sido atacada por $\mathbf{T}$. heveae.

\section{CONCLUSÃO}

Das 33 progênies testadas, oito apresentaram resistência por não preferência e/ou antibiose ou então por tolerância a pelo menos uma das espécies de ácaros: FRP/12110, FRP/924, FRP/1611, FRP/832, FRP/3511, FRP/2821, FRP/3012 e FRP/3523.

\section{AGRADECIMENTO}

Os autores agradecem à Fundação de Amparo à Pesquisa do Estado de São Paulo (FAPESP), pelo financiamento do projeto e pela bolsa de iniciação científica concedida ao segundo autor.

\section{REFERÊNCIAS}

CORTEZ, J.V.; MARTIN, N.B. A sazonalidade da produção da seringueira e a política brasileira de contingenciamento da importação de borracha natural. Informações Econômicas, v.26, n.7, p.45-54, 1996.

DAUD, R.D.; FERES, R.J.F. Dinâmica populacional de ácaros fitófagos (Acari, Eriophyidae, Tenuipalpidae) em seis clones de seringueira no sul do Estado de Mato Grosso. Revista Brasileira de Entomologia, v.51, n.3, p.377-381, 2007. Disponível em: <http://www.scielo.br/scielo.php?script=sci_arttext\&pid=S0085$56262007000300016 \& l n g=p t \& n r m=i s o \& t l n g=p t>$. Acesso em 20 abr. 2009. doi: 10.1590/S0085-56262007000300016.

FERLA, N.J. Ecologia e controle de ácaros (Acari) da seringueira (Hevea brasiliensis Muell. Arg.) no Estado de Mato Grosso. 2001. 141f. Tese (Doutorado em Entomologia) - Escola Superior de Agricultura “Luiz de Queiroz”, Universidade de São Paulo, Piracicaba - SP.

FERLA, N.J.; MORAES, G.J. Ciclo biológico de Calacarus heveae Feres, 1992. Revista Brasileira de Entomologia, v.47, n.3, p.399-402, 2003. Disponível em: <http:// www.scielo.br/scielo.php?script=sci_arttext\&pid=S0085$56262003000300001 \& \operatorname{lng}=\mathrm{en} \& \mathrm{nrm}=\mathrm{iso} \& \mathrm{t} \operatorname{lng}=\mathrm{pt}>$. Acesso em 20 abr. 2009. doi: 10.1590/S0085-56262003000300006.

FURLANI, R.C.M. Caracterização de progênies de seringueira [Hevea brasiliensis (Willd. Ex Adr. de Juss.) Müell. Arg.] na região de Ilha Solteira - SP. 2001. 113f. Dissertação (Mestrado em Agronomia) - Universidade Estadual Paulista (UNESP), Ilha Solteira, SP.

FURQUIM, G.V. Flutuação populacional de ácaros e caracterização de sintomas de Calacarus heveae em clones de seringueira (Hevea brasiliensis Müell. Arg.) cultivados em Jaboticabal - SP. 1994. 33f. Monografia (Trabalho de graduação em Agronomia) - Universidade Estadual Paulista (UNESP), Jaboticabal, SP.

HERNANDES, F.A.; FERES, R.J.F. Review about mites (Acari) of rubber trees (Hevea spp., Euphorbiaceae) in Brazil. Biota Neotropica, v.6, n.1, 2006. Disponível em: <http:// w w w. bi o t a n e t r o p i c a o r g. br/v $6 \mathrm{n} 1 / \mathrm{pt} /$ abstract?article+bn00406012006>. Acesso em: 16 setembro 2008 .

GONÇALVES, P.S. Recomendação de clones de seringueira para o Estado de São Paulo. In: CICLO DE PALESTRAS SOBRE A HEVEICULTURA PAULISTA, Barretos, 1998. Anais... Barretos: Secretaria de Agricultura e Abastecimento do Estado de São Paulo; Associação Paulista de Produtores e Beneficiadores de Borracha (APABOR), 1999. p.115140 .

GONÇALVES, P.S. et al. Manual de heveicultura para o Estado de São Paulo. Campinas: Instituto Agronômico, 2001. 78 .

LARA, F.M. Princípios de resistência de plantas a insetos. 2.ed. São Paulo: Ícone, 1991. 336p.

PONTIER, K.J.B. et al. Biology of Tenuipalpus heveae (Acari, Tenuipalpidae) on rubber tree leaves. Acarologia, v.41, n.4, p.423-427, 2000.

SABELIS, M.W.; BRUIN, J. Evolutionary ecology: life history patterns, food plant choice and dispersal. In: LINDQUIST, E.E. et al. (Ed.). Eriophyoid mites: their biology, natural enemies and control. Amsterdam: Elsevier, 1996. p.329366. (World Crop Pests, 6).

TADMOR, Y. et al. Antibiosis of maize inbred lines to the carmine spider mite, Tetranychus cinnabarinus. Phytoparasitica v.27, n.1, p.35-41, 1999.

Ciência Rural, v.39, n.7, out, 2009. 
VIEIRA, M.R.; GOMES, E.C. Sintomas, desfolhamento e controle de Calacarus heveae Feres, 1992 (Acari: Eriophyidae) em seringueira. Cultura Agronômica, v.8, n.1, p.53-71, 1999.

VIEIRA, M.R.; GOMES, E.C. Ácaros da seringueira: sintomas e controle. In: CICLO DE PALESTRAS SOBRE HEVEICULTURA PAULISTA, 3., 2002, São José do Rio Preto, SP. Anais... São José do Rio Preto: Secretaria de Agricultura e Abastecimento do Estado de São Paulo; Associação Paulista de Produtores e Beneficiadores de Borracha (APABOR), 2003. p.63-72.
VIEIRA, M.R. et al. Sintomatologia do ataque de Calacarus heveae Feres, 1992 (Acari: Eriophyidae) em seringueira (Hevea brasiliensis Müell. Arg.). Revista de Agricultura, v.75, n.3, p.405-414, 2000.

VIEIRA, M.R. et al. Resistência de cultivares de algodoeiro ao ácaro branco Polyphagotarsonemus latus (Banks) (Acari: Tarsonemidae). 1. Experimentos em laboratório. Revista Brasileira de Oleaginosas e Fibrosas, v.6, n.2, p.545-555, 2002. Disponível em: <http://www.cnpa.embrapa.br/rbof/ fasciculos.php>. Acesso em: 20 abr. 2009. 